\title{
Antecedentes biológicos de hormigas presentes en Chile publicados en revistas científicas nacionales y extranjeras durante el siglo $\mathrm{XX}$
}

\author{
Biological background of ants found in Chile published in national and foreign \\ scientific journals during the XX century
}

\begin{abstract}
HUGO TORRES-CONTRERAS
Departamento de Ciencias Ecológicas, Facultad de Ciencias, Universidad de Chile, Casilla 653, Santiago, Chile, e-mail: htoresco@icaro.dic.uchile.cl
\end{abstract}

\begin{abstract}
RESUMEN
Se revisa la información sobre biología de hormigas presentes en Chile publicada en revistas científicas nacionales y extranjeras durante el período 1900-1999. Se evalúan estos antecedentes en términos de: (i) la dinámica temporal de la producción de publicaciones, (ii) las principales temáticas de estudio, y (iii) los géneros de formícidos en los cuáles se ha concentrado la investigación. Los datos recolectados muestran que sólo a partir de la década del 60’ se produce un marcado crecimiento del número de artículos sobre hormigas, lo cual alcanza su máxima expresión durante la década del 90'. Los principales tópicos de investigación han sido, en orden decreciente, aspectos sobre taxonomía, uso del hábitat, períodos de actividad, conductas tróficas y de reconocimiento entre individuos. Los géneros de formícidos en los que se han centrado estos estudios corresponden a Camponotus, Solenopsis, Pogonomyrmex, Brachymyrmex, Conomyrma y Linepithema. Estos resultados son discutidos a la luz de acontecimientos históricos que habrían determinado el curso de las investigaciones. Finalmente, se proponen líneas de investigación que en el futuro podrían desarrollar los estudios sobre hormigas en Chile.
\end{abstract}

Palabras clave: hormigas, Chile, ecología, taxonomía, Camponotus, Solenopsis.

\begin{abstract}
Information is reviewed on the biology of ants found in Chile published in national and foreign scientific journals during the period 1900-1999. The information is evaluated in terms of: (i) temporal dynamics of publication production, (ii) main themes of study, and (iii) formicid groups in which the investigation has been concentrated. The collected data show that in the decade the 60's, the number of articles on ants grew, reaching a peack during the 90's. The main topics of investigation have been, in decreasing order, aspects of taxonomy, habitat use, periods of activity, trofic behavior and inter-individuals recognition. The formicid groups in which these studies have been focused correspond to Camponotus, Solenopsis, Pogonomyrmex, Brachymyrmex, Conomyrma and Linepithema. These results are discussed in the light of historical events that may have determined the course of research. Finally, future lines of investigation for the studies of ants in Chile are proposed.
\end{abstract}

Key words: ants, Chile, ecology, taxonomy, Camponotus, Solenopsis.

\section{INTRODUCCIÓN}

Existen aproximadamente 8.800 especies conocidas de hormigas en el mundo, las cuales dominan una variedad de hábitats y ambientes ecológicos, donde alcanzan una gran abundancia (Holldobler \& Wilson 1990). Las hormigas constituyen el principal grupo de insectos eusociales, los cuáles se caracterizan por presentar: (i) individuos adultos que cooperan en la construcción del nido y el cuidado de la progenie, (ii) división de la labor reproductiva, con individuos estériles que trabajan a favor de la fecundidad del nido, y (iii) sobreposición de generaciones, las cuáles son capaces de contribuir a las tareas de la colonia (Seger 1991, Bourke 1997). Una característica distintiva de las hormigas es que presentan tres castas morfológicas de sexo femenino - obreras, soldados y reinas - y una casta de individuos machos que cumple una breve función reproductiva durante el vuelo nupcial (Wilson 1971, Oster \& Wilson 1978). En una colonia, la casta obrera es la que desempeña una mayor variedad de tareas como por ejemplo, forrajeo, cuidado de progenie y construcción de nidos (Wilson 1985, Gordon 1996).

Otra característica importante de las hormigas es su haplodiploidía, debido a lo cual los huevos no 
fertilizados se desarrollan en machos haploides y los huevos fertilizados en hembras diploides (Heinrich 1978, Brockman 1984). El modo en que se determina el sexo favorece la selección por parentesco, en la cual la proporción de genes compartidos por las obreras condiciona el cuidado de la progenie de sus hermanas (altruismo reproductivo), de forma tal que aseguran indirectamente a través de sus parientes una mayor representación o inversión futura de sus genes en la población (fitness inclusivo), en comparación a si estos individuos produjeran su propia descendencia (Hamilton 1964, Trivers \& Hare 1976, Krebs \& Davies 1993). Existen además una serie de atributos como por ejemplo, fundación de colonias a partir de una o más reinas, comunicación química (feromonas) entre obreras, movimientos de forrajeo en solitario y/o en columna, y relaciones simbióticas con otras especies de hormigas, insectos y plantas, que convierten a las hormigas en interesantes objetos de estudio (Holldobler \& Wilson 1990).

En Chile existen 62 especies de hormigas, las cuáles se distribuyen en 22 géneros, los que a su vez están adscritos a cinco subfamilias (Ponerinae, Pseudomyrmecinae, Myrmicinae, Dolichoderinae, Formicinae) (Snelling \& Hunt 1975, Rojas \& Elgueta 1995). La riqueza de formícidos en Chile es pobre en comparación a la fauna mirmecológica de Sudamérica, incluye 10 especies consideradas como hormigas introducidas, cuatro con distribución neotropical, 14 con distribución patagónica, y 34 especies endémicas (modificado de Snelling \& Hunt 1975). Sólo en las últimas dos décadas se han realizado trabajos que han abordado en profundidad aspectos de la ecología de las hormigas presentes en Chile; sin embargo, existe una escasez de conocimiento en muchas temáticas. El objetivo de este trabajo fue recopilar información relevante sobre la biología de hormigas en Chile presente en revistas científicas extranjeras y - especialmentenacionales durante el período 1900-1999. Se analiza esta información en términos de: (i) la dinámica temporal de la producción de publicaciones, (ii) las principales temáticas de estudio, y (iii) los géneros de formícidos en los cuáles se ha concentrado la investigación. Los resultados son discutidos a la luz de acontecimientos históricos que habrían determinado el curso de las investigaciones. Finalmente, se proponen líneas de investigación que en el futuro podrían desarrollar los estudios sobre hormigas presentes en Chile.

\section{MATERIALES Y MÉTODOS}

Se revisaron los catálogos de revistas científicas nacionales publicados por M. Etcheverry en la
Revista Chilena de Historia Natural a partir de 1985, y se actualizó y completó la búsqueda bibliográfica hasta el año 1999 inclusive. Las principales revistas consultadas fueron: (1) Acta Entomológica (1985-1999), (2) Actas de la Societé Scientifique du Chili (1891-1971), (3) Anales de la Academia Chilena de Ciencias Naturales (19261968), (4) Anales del Museo de Historia Natural de Valparaíso (1968-1999), (5) Anales del Instituto de la Patagonia (Ciencias Naturales) (19701999), (6) Anales del Museo Nacional de Historia Natural (1891-1910), (7) Anales de Zoología Aplicada (1914-1922), (8) Boletín del Museo Nacional de Historia Natural (1908-1999), (9) Boletín del Museo Regional de Concepción (19261999), (10) Boletín de la Sociedad de Biología de Concepción (1927-1999), (11) Bosque (19751998), (12) Gayana (Zoología) (1961-1999), (13) Investigaciones Zoológicas Chilenas (1950-1964), (14) Medio Ambiente (1975-1996), (15) Noticiario Mensual del Museo Nacional de Historia Natural (1956-1999), (16) Publicaciones del Centro de Estudios Entomológicos (1960-1974), (17) Publicación Ocasional del Museo Nacional de Historia Natural (1963-1992), (18) Revista Chilena de Entomología (1951-1999), y (19) Revista Chilena de Historia Natural (1897-1999).

En relación a las publicaciones sobre hormigas en Chile presentes en revistas científicas extranjeras, se realizó una búsqueda bibliográfica en la base de datos BIOSIS durante el período comprendido entre 1985-2000. En forma paralela, esta búsqueda fue complementada con la base de datos CAB Abstracts (INIA), lo cual permitió cubrir los años 1972-2000. En ambos casos se utilizaron como palabras claves, términos lo más amplios y generales posibles (e.g., Hymenoptera, ant(s), Chile). Es importante mencionar que tanto en el caso de publicaciones nacionales y extranjeras, parte de la información - en especial aquella referida a trabajos anteriores a la década del 70' - fue obtenida a partir de una minuciosa revisión de la bibliografía y/o referencias citada en los trabajos de que se disponía. Los nombres taxonómicos de las especies de hormigas mencionadas en la sección resultados y discusión han sido actualizados según el catálogo de Snelling \& Hunt (1975) y corroborados con la reciente revisión de Rojas \& Elgueta (1995); entre este lapso de tiempo destaca la nueva sinonimia del género Conomyrma Forel (= Araucomyrmex Gallardo) propuesta por Snelling (1981) y la nueva combinación de Linepithema humile (Mayr) (= Iridomyrmex humilis) propuesta por Shattuk (1992). En resultados y discusión me referiré en forma general a hormigas chilenas; este término agrupa tanto a las especies nativas como a las 
introducidas (estas últimas serán destacadas con un asterisco $(*)$ en el texto, figuras y tabla).

\section{RESULTADOS}

Las primeras informaciones respecto a hormigas chilenas, se remontan a mediados del siglo XIX y corresponden esencialmente a antecedentes taxonómicos. El trabajo pionero de Spinola (1851) publicado en la clásica obra "Historia física y política de Chile" de C. Gay documenta la existencia de siete especies de formícidos (Camponotus distinguendus, C. chilensis, C. ovaticeps, Lasiophanes nigriventris, Pseudomyrmex lynceus, Solenopsis gayi, y Pogonomyrmex bispinosus). Posteriormente, el trabajo de Berg (1890) recopila antecedentes documentados por autores como A. Forel, F. Smith, J. Roger y esencialmente G. Mayr, reportando la presencia de 23 especies así como algunos comentarios sobre la biología de estas especies. A similar resultado llega un trabajo de Emery (1894) donde se describen dos nuevas especies (Brachymyrmex giardii y B. laevis), posteriormente este mismo autor (Emery 1895) documenta a cinco nuevas especies (Nothidris latastei, Solenopsis germaini, S. helena, S. latastei, y Conomyrma minutus) y sus lugares de recolección.

\section{Dinámica temporal de producción de publicacio- nes}

Durante el siglo XX existen 58 publicaciones que entregan antecedentes biológicos sobre la fauna mirmecológica de Chile. A continuación se detalla de manera parcelada el curso seguido por la producción de artículos sobre hormigas chilenas durante las diferentes décadas (Fig. 1). En esta cuantificación no se incluyen trabajos referentes a biología de hormigas chilenas presentes en islas oceánicas, debido a la escasa información existente respecto al tema (Wheeler 1923, Kuschel 1957, Campos \& Peña 1973, Peña 1987). Por otra parte, cuando es pertinente se hace mención a antecedentes sobre hormigas foráneas publicados en revistas científicas nacionales.

Período 1900-1909. En este período no existe información sobre hormigas chilenas. Sin embargo, Gallardo (1908) publica un artículo en la Revista Chilena de Historia Natural sobre la fundación de colonias de "hormigas sauvá y negra" (Atta sp.), y su alimentación y mantención a partir de cultivo de hongos.

Período 1910-1919. En este período tampoco existe información sobre hormigas chilenas. A pesar de esto, se publican en revistas nacionales tres pequeñas notas sobre cultivo de hongos, daño a cultivos agrícolas y vida en simbiosis con orugas, por parte de hormigas foráneas (Porter 1910, La Redacción 1914, Porter 1915).

Período 1920-1929. Durante este período existen dos artículos en los que se hace mención a hormigas chilenas. Una publicación se refiere a la presencia de formícidos (Tetramorium caespitum*, Lasiophanes valdiviensis, Brachymyrmex giardii) en la región mediterránea y sur de Chile (Santschi 1922), y la otra a la diagnosis de especies (Solenopsis gayi, S. patagonica, Tapinoma antarcticum) reportadas también por Santschi en estas mismas regiones (Porter 1923b). En forma paralela, se publican en revistas nacionales tres pequeñas notas sobre parasitismo de colmenas de abejas, coexistencia con bracónidos y depredación de piojos, por parte de hormigas foráneas (Wolffsohn 1920, Porter 1923a, 1926).

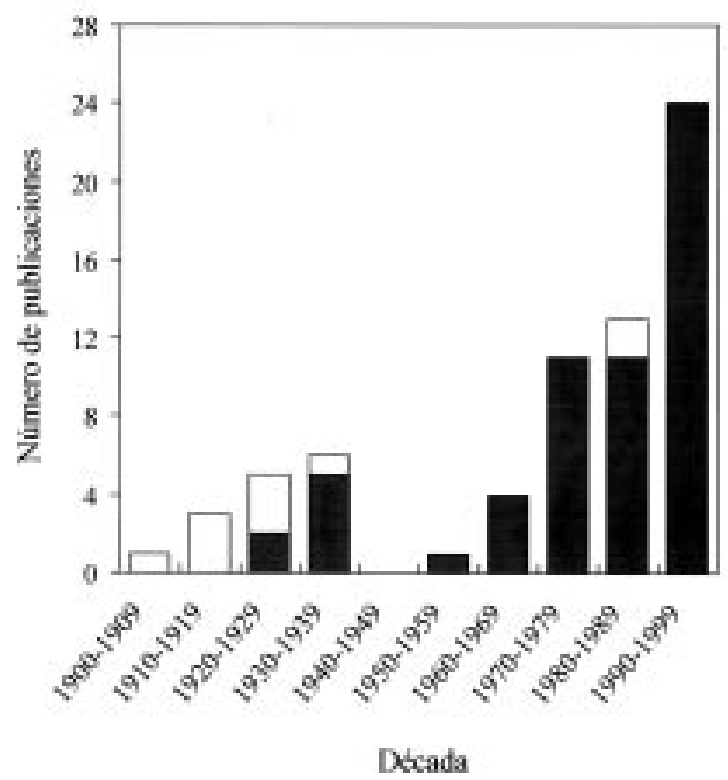

Fig. 1: Dinámica temporal de la producción de publicaciones sobre formícidos chilenos durante el siglo XX. La barra blanca corresponde a información sobre hormigas foráneas divulgada en revistas científicas nacionales, y la barra oscura a antecedentes sobre especies presentes en Chile publicados tanto en revistas nacionales como extranjeras; la suma de ambas barras representa el total de publicaciones por década.

Temporal dynamics of the publication production on Chilean formicids during the XX century. The white bar corresponds to information on foreign ants published in national journals, and the dark bar to information of species found in Chile published both in national and foreign journals; the sum of both bars represents the total of publications for decade. 
Período 1930-1939. Durante este período se produjeron cinco artículos sobre hormigas chilenas. Un par de publicaciones analiza la comunicación química y conductas asociadas de exploración y orientación del movimiento en obreras de Solenopsis gayi y Pogonomyrmex bispinosus (Goetsch 1930a, 1930b). Por otra parte, tres trabajos entregan antecedentes taxonómicos sobre el número de formícidos presentes en Chile. Es así como Goetsch (1933) documenta la existencia aproximada de 34 especies, y el trabajo de Menozzi (1935) y su posterior traducción al alemán por parte de Goetsch \& Menozzi (1935) reportan la existencia de 56 especies, destacando el registro de tres nuevas especies (Conomyrma goetschi, Myrmelachista goetschi y Camponotus hellmichi). Finalmente, un artículo publicado en los Anales de la Academia Chilena de Ciencias Naturales documenta procedimientos para exterminar hormigas y evitar así los daños provocados en la agricultura (Pérez-Canto 1937).

Período 1940-1949. De acuerdo a la búsqueda y revisión efectuada en este trabajo, durante esta década no se registran publicaciones que contengan información relevante sobre hormigas chilenas, ni tampoco antecedentes generales sobre formícidos foráneos que hayan sido documentados en revistas científicas nacionales.

Período 1950-1959. El trabajo de Kusnezov (1959) corresponde al único antecedente publicado durante esta década, que hace mención a hormigas chilenas. Este artículo trata aspectos taxonómicos de formícidos que se distribuyen en el oeste de la Patagonia y Tierra del Fuego, e incluye importantes comentarios acerca de la biología de estas especies. Específicamente, este autor documenta para esta región hormigas pertenecientes a los géneros Solenopsis (S. patagonica), Antichthonidris (A. bidentatus, A. denticulatus), Pogonomyrmex ( $P$. angustus, $P$. laevigatus, $P$. odoratus), Conomyrma (C. antarcticus, C. tener), Lasiophanes (L. nigriventris, L. picinus) y Camponotus (C. chilensis, C. distinguendus), los cuáles se presentan esencialmente asociados a bosques de Nothofagus, coníferas (araucarias, cipreces de las guaitecas), alerces y mañios.

Período 1960-1969. En este período se producen cuatro trabajos sobre hormigas de Chile. De éstos, tres corresponden a temáticas taxonómicas, destacando el trabajo sobre zoogeografía de hormigas Sudamericanas de Kusnezov (1963), quien basado en el trabajo de Goetsch \& Menozzi (1935) y colecciones de T. Cekalovic en el extremo sur del país (provincia de Magallanes), dedica una sección a la fauna mirmecológica de Chile. En este trabajo el autor determina un total aproximado de 43 especies, entrega antecedentes sobre las localidades de recolección, compara su diversidad y riqueza con las faunas de Norte América, Patagonia, Australia y Nueva Zelandia, y discute los tiempos de evolución y posibles centros de origen de las especies. El otro par de publicaciones abordan aspectos taxonómicos de los géneros Pogonomyrmex (específicamente de $P$. vermiculatus en la región de Magallanes, Cekalovic 1964), y Nothidris (esencialmente de $N$. bicolor en la región de Coquimbo, Ettershank 1965). Finalmente, Ipinza-Regla (1969) discute el papel de Solenopsis gayi como modificador del paisaje correspondiente a la sabana espinosa chilena.

Período 1970-1979. Durante este período se publicaron 11 artículos sobre hormigas chilenas. Entre éstos, destacan el trabajo de Kempf (1970, 1972) que corresponde a la elaboración de un catálogo de formícidos chilenos en el cual se reportan 52 especies, y el documento de Snelling \& Hunt (1975) el cual consiste en una clave taxonómica para la identificación de 62 especies de hormigas presentes en Chile, y contiene además valiosos datos sobre sus rangos de distribución y comentarios acerca de su biología. Previo a este documento, un par de trabajos había documentado el patrón temporal de actividad en las especies competidoras Conomyrma antarcticus y Tapinoma antarcticum (Hunt 1974), y el reporte de las siguientes nuevas especies: Nothidris cekalovici, Solenopsis dysderces, Dorymyrmex agallardoi, Conomyrma hunti, C. hypocritus, $C$. incomptus, C. pappodes, y C. pogonius (Snelling 1975). Finalmente, durante estos años también se publicaron algunos artículos sobre la generación de cuadros anafilácticos por la "picadura" de Heteroponera carinifrons (Ipinza \& Capurro 1972, Ipinza \& Schenone 1972), abundancia de formícidos en la sabana espinosa en relación a variables abióticas (Ipinza-Regla 1971a, 1971b), y la presencia de Solenopsis gayi en la dieta de Pitius pitius (Ave, Picidae) (Moroni 1977) y de especies de Camponotus en la dieta de Liolaemus monticola (Reptilia, Iguanidae) (Fuentes \& Ipinza 1979).

Período 1980-1989. Durante esta década también se produjeron 11 artículos sobre hormigas chilenas, pero además se publicaron en revistas nacionales dos trabajos sobre formícidos foráneos, uno relacionado con hormigas cortadoras de hojas paraguayas y el otro referente a comunidades de hormigas del mato grosso brasileño (Fowler 1981, 1988). En lo que respecta a la información nacional, destacan los trabajos sobre distribución de especies de formícidos - de las subfamilias Mirmicinae y Dolichoderinae - en relación a los tipos de hábitats y condiciones bioclimáticas que 
ocupan (Ipinza-Regla \& Covarrubias 1982, 1989), y distribución espacial de nidos de hormigas en gradientes altitudinales de sectores precordilleranos de Chile central (Ipinza-Regla et al. 1983, 1986, Covarrubias et al. 1987). Por otra parte, en este período también se documentó información sobre el efecto de depredadores, conductas tróficas y períodos de actividad de Camponotus morosus (Ipinza-Regla 1985, Grez et al. 1986, Ipinza-Regla et al. 1988), así como el rol desempeñado como vector de infecciones bacteriológicas intrahospitalarias y vector de contaminación microbiana en industrias de alimentos, por parte de Linepithema humile* (IpinzaRegla et al. 1981, 1984). Finalmente, un trabajo discute el efecto perjudicial indirecto que Linepithema humile* ocasiona a cultivos agrícolas (Ripa 1987).

Período 1990-1999. Esta década constituye sin duda la etapa más productiva de publicaciones sobre hormigas chilenas, registrándose un total de 24 artículos. Entre estos trabajos destacan los estudios sobre ensambles de hormigas granívoras (Conomyrma goetschi, Brachymyrmex giardii, Solenopsis gayi, y Pogonomyrmex vermiculatus), donde se discute su efecto en la generación de patrones y procesos a nivel comunitario (Medel \& Vásquez 1994, Medel 1995, Medel \& Fuentes 1995, Vásquez et al. 1995, Fuentes et al. 1996). Durante este período también se analizó el nivel de "hermetismo" que presentan sociedades homo y/o heteroespecíficas de hormigas (Camponotus morosus, C. chilensis, Brachymyrmex giardii, Solenopsis gayi), determinándose el tiempo de latencia y orden en que se presentan ciertas unidades conductuales (e.g., exploración antenal, abertura mandibular, flexión del abdomen) que permiten el reconocimiento y aceptación entre individuos (Ipinza-Regla et al. 1991, 1993b, 1994, 1996, 1998, Ipinza-Regla \& Morales 1998). En un siguiente orden de importancia, destacan los trabajos relacionados con problemáticas de uso del hábitat por parte de distintas especies de hormigas; específicamente asociación a diferentes tipos de hábitats (Solervicens et al. 1991, Lewis \& Peredo 1993), capacidad de recuperación poblacional frente a una perturbación del hábitat (Saiz \& Carvajal 1990), y distribución espacial de nidos (Ipinza-Regla et al. 1990). Por otra parte, algunos artículos documentan el efecto negativo de Linepithema humile* y Solenopsis gayi sobre árboles frutales (Ripa et al. 1993a, 1993b, Larraín et al. 1995, Ripa et al. 1998). Además, un trabajo evaluó la conducta de "atención" de áfidos por parte de Linepithema humile* cuando ésta es expuesta a compuestos volátiles (semioquímicos) involucrados en la conducta de espaciamiento de estos áfidos (Córdova-Yamauchi et al. 1998). Finalmente, para especies pertenecientes al género cosmopolita Camponotus se documentan antecedentes acerca de sus conductas tróficas (RíosAramayo \& Jiménez 2000), ámbito de hogar (Ipinza-Regla et al. 1993a), genética de colonias (Eaton \& Medel 1994), y notas técnicas (IpinzaRegla et al. 1992).

\section{Principales temáticas de estudio}

Los principales tópicos de investigación sobre hormigas chilenas desarrollados durante el siglo XX se muestran en la Fig. 2 y Tabla 1. Es importante mencionar que una publicación puede haber analizado más de una temática, y por ende ésta fue considerada un aporte de antecedentes en varias categorías de estudio. Entre la totalidad de artículos, destacan claramente los trabajos sobre taxonomía, los cuáles registraron una producción de 10 publicaciones durante el período 19001999 (Porter 1923, Goetsch 1933, Kusnezov 1959, 1963, Cekalovic 1964, Ettershank 1965, Kempf 1970, 1972, Snelling 1975, Snelling \& Hunt 1975).

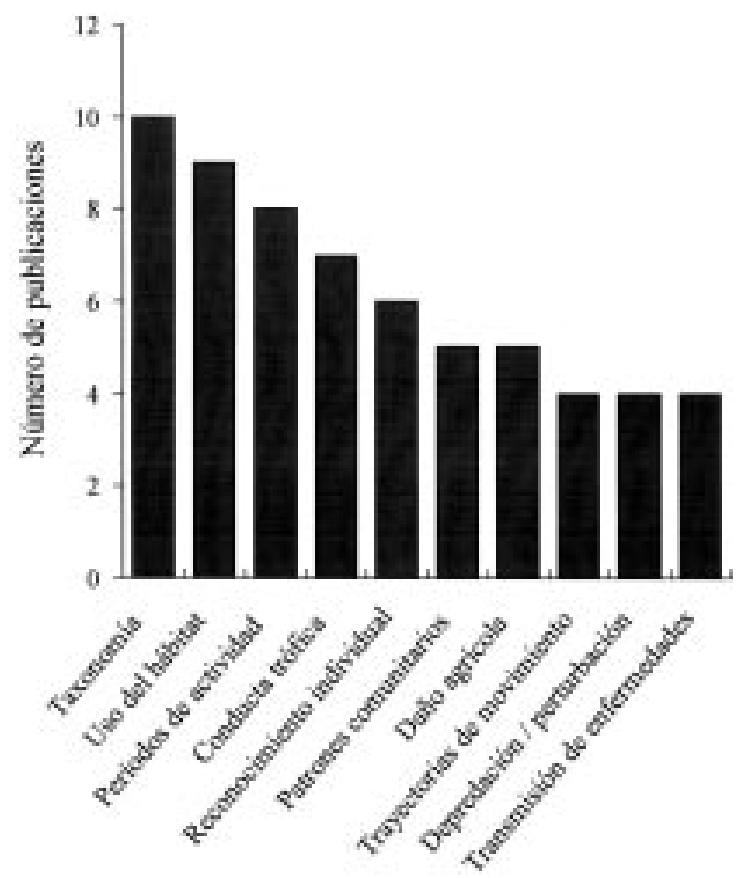

Temática de estudio

Fig. 2: Principales temáticas de estudio sobre hormigas chilenas, aparecidas en publicaciones nacionales y extranjeras durante el período 19001999.

Main themes of study on Chilean ants, published in national and foreign journals during the period 1900-1999. 


\section{TABLA 1}

Principales trabajos sobre biología de hormigas chilenas. Para cada artículo se detalla la línea de investigación analizada, los géneros de hormigas estudiados, y un comentario sobre su contenido. Se excluyen seis trabajos sobre taxonomía que corresponden a listas y/o catálogos de especies (Goetsch 1933, Kusnezov 1959, 1962, Kempf 1970, 1972, Snelling \& Hunt 1975). Los símbolos corresponden a especies: $*=$ introducidas, ${ }^{\mathrm{N}}=$ con distribución neotropical, ${ }^{\mathrm{P}}=$ con distribución patagónica, ${ }^{\mathrm{E}}=$ endémicas

Main studies about the biology of Chilean ants. For each article, I show the theme of investigation, the ant genera studied, and comments on the results. Six papers on taxonomy that correspond to lists and/or catalogues of species have been excluded (Goetsch 1933, Kusnezov 1959, 1962, Kempf 1970, 1972, Snelling \& Hunt 1975). The symbols represent species: ${ }^{*}=$ introduced, ${ }^{\mathrm{N}}=$ with distribution neotropical, ${ }^{\mathrm{P}}=$ with distribution patagonica, ${ }^{\mathrm{E}}=\mathrm{endemics}$

\begin{tabular}{|c|c|c|}
\hline Referencia & Temática estudiada & Especie analizada \\
\hline Cekalovic (1964) & Taxonomía & Pogonomyrmex vermiculatus $\mathrm{P}$ \\
\hline Córdova-Yamauchi et al. (1998) & Comunicación química & Linepithema humile* \\
\hline Covarrubias et al. (1987) & Uso del hábitat & 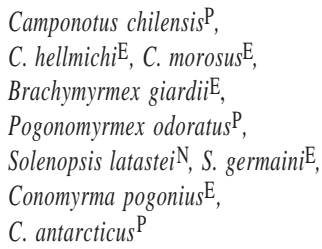 \\
\hline Eaton \& Medel (1994) & Genética de colonias & Camponotus chilensis ${ }^{\mathrm{P}}$ \\
\hline
\end{tabular}

Observaciones

Se documenta la presencia de esta especie en la región de Magallanes

Se evaluó la conducta de "atención" de áfidos por parte de esta especie, cuando es expuesta a compuestos volátiles (semioquímicos) involucrados en la conducta de espaciamiento de estos áfidos

Se establecen correlaciones entre gradiente altitudinal, cobertura vegetal, pH suelo, y número de especies, número de nidos de hormigas

Se infiere la estructura reproductiva (fundación monogínica y/o poligínica del nido) y el grado de parentesco en obreras de diferentes colonias

\begin{tabular}{|c|c|c|}
\hline Ettershank (1965) & Taxonomía & Nothidris bicolor $^{\mathrm{E}}$ \\
\hline Fuentes \& Ipinza (1979) & Depredación/Perturbación & $\begin{array}{l}\text { Camponotus chilensis }{ }^{\mathrm{P}} \text {, } \\
\text { C. morosus }{ }^{\mathrm{E}}, \text { C. hellmichi } \\
\text {, } \\
\text { C. } \text { ovaticeps }^{\mathrm{E}}\end{array}$ \\
\hline Fuentes et al. (1996) & $\begin{array}{l}\text { Períodos de actividad, conductas } \\
\text { tróficas, patrones comunitarios }\end{array}$ & $\begin{array}{l}\text { Solenopsis gayi }{ }^{\mathrm{E}}, \\
\text { Conomyrma goetschi }^{\mathrm{E}} \text {, } \\
\text { Brachymyrmex giardii }^{\mathrm{E}}\end{array}$ \\
\hline Goetsch (1930a, 1930b) & Trayectorias de movimiento & $\begin{array}{l}\text { Solenopsis gayi }{ }^{\mathrm{E}}, \\
\text { Pogonomyrmex bispinosus } \mathrm{E}\end{array}$ \\
\hline Grez et al. (1986) & Conductas tróficas & Camponotus morosus $\mathrm{E}$ \\
\hline Hunt (1974) & Períodos de actividad & $\begin{array}{l}\text { Conomyrma antarcticus }{ }^{\mathrm{P}} \text {, } \\
\text { Tapinoma antarcticum } \\
\mathrm{E}\end{array}$ \\
\hline Ipinza-Regla $(1969,1971 a)$ & Períodos de actividad & $\begin{array}{l}\text { Solenopsis gayi }{ }^{\mathrm{E}}, \mathrm{y} \\
\text { formícidos en general }\end{array}$ \\
\hline
\end{tabular}

Se realiza el diagnóstico de esta especie en la región de Coquimbo

Se analiza la presencia de formicídos en el contenido estomacal de Liolaemus monticola (Reptilia) de Chile, se analizan: actividad de hormigas en el rango diario de temperaturas del suelo, sobreposición en utilización de cebos, y correlaciones entre precipitaciones y abundancias relativas de las especies

Se estudian las conductas de exploración y orientación de los movimientos exhibidos por obreras entre el nido y una fuente de alimento

Se estudia la dieta de esta especie en el matorral de Chile central durante la estación de verano

Se analiza el efecto de la temperatura superficial del suelo sobre las actividades de forrajeo, y la coexistencia de estas especies

Se analiza el efecto de la temperatura y humedad relativa, sobre la abundancia y período de actividad de las hormigas
En localidades de la región árida y semiárida 
TABLA 1 (continuación)

\begin{tabular}{|c|c|c|c|}
\hline Referencia & Temática estudiada & Especie analizada & Observaciones \\
\hline Ipinza-Regla (1971b) & Uso del hábitat & $\begin{array}{l}\text { Linepithema*, Dorymyrmex }^{\mathrm{E}} \text {, } \\
\text { Tapinoma }^{\mathrm{E}} \text {, Solenopsis, } \\
\text { Pogonomyrmex, Brachymyrmex }^{\mathrm{E}} \text {, } \\
\text { Myrmelachista, Camponotus }\end{array}$ & $\begin{array}{l}\text { Se documenta la abundancia de la fauna } \\
\text { mirmecológica en el estrato árboreo-arbustivo de } \\
\text { la sabana espinosa central (Curacaví, V región) }\end{array}$ \\
\hline Ipinza-Regla (1985) & Depredación/Perturbación & $\begin{array}{l}\text { Camponotus morosus } \mathrm{S}^{\mathrm{E}}, \text { C. chilensis } \mathrm{P} \text {, } \\
\text { Brachymyrmex giardil }^{\mathrm{E}} \text {, } \\
\text { Myrmelachista hoffmanni } \mathrm{E} \text {, } \\
\text { Pogomoyrmex bispinosus }{ }^{\mathrm{E}} \text {, } \\
\text { P. angustus }{ }^{\mathrm{P}} \text {, Tetramorium } \\
\text { caespitum }^{*} \text {, Linepithema humile* }\end{array}$ & $\begin{array}{l}\text { Se determina la presencia de formícidos en el } \\
\text { contenido gástrico de Liolaemus } \\
\text { monticola (Reptilia) }\end{array}$ \\
\hline $\begin{array}{l}\text { Ipinza \& Capurro (1972), } \\
\text { Ipinza \& Schenone (1972) }\end{array}$ & Transmisión de enfermedades & Heteroponera carinifrons ${ }^{\mathrm{E}}$ & $\begin{array}{l}\text { Se documentan cuadros anafilácticos en la región } \\
\text { de Antofagasta provocados por la "picadura" de } \\
\text { esta especie }\end{array}$ \\
\hline Ipinza-Regla \& Covarrubias (1982) & Uso del hábitat & 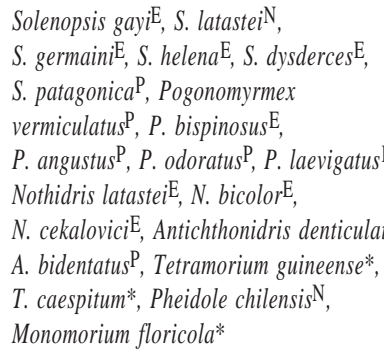 & $\begin{array}{l}\text { Se analiza la distribución de estas especies } \\
\text { (subfamilia Myrmicinae), en relación al tipo de } \\
\text { hábitat y condición bioclimática que utilizan } \\
{ }_{s} \mathrm{P} \\
{ }_{\text {atus }}{ }^{\mathrm{P}} \text {, }\end{array}$ \\
\hline Ipinza-Regla \& Covarrubias (1989) & Uso del hábitat & 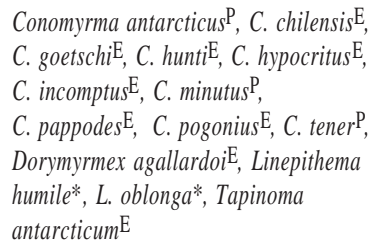 & $\begin{array}{l}\text { Se analiza la distribución de estas especies } \\
\text { (subfamilia Dolichoderinae) de acuerdo a su } \\
\text { presencia en diferentes regiones bioclimáticas }\end{array}$ \\
\hline
\end{tabular}

Ipinza-Regla \& Morales (1998) Reconocimiento entre individuos Camponotus morosus ${ }^{\mathrm{E}}$

Ipinza-Regla et al. (1981)

Ipinza-Regla et al. (1983)

Ipinza-Regla et al. (1984)

Ipinza-Regla et al. (1986)

Ipinza-Regla et al. (1988)

Ipinza-Regla et al. (1990)
Uso del hábitat

Transmisión de enfermedades

Uso del hábitat

Transmisión de enfermedades

Uso del hábitat

Períodos de actividad

Camponotus morosus ${ }^{\mathrm{E}}$

Brachymyrmex giardii ${ }^{\mathrm{E}}$, Camponotus morosus $\mathrm{E}$, Solenopsis gayi $\mathrm{E}$, Conomyrma hypocritus $\mathrm{E}$

Camponotus chilensis ${ }^{\mathrm{P}}$, C. hellmichi $\mathrm{E}$, Pogonomyrmex odoratus ${ }^{\mathrm{P}}$, Solenopsis lataste $^{\mathrm{N}}{ }^{\mathrm{N}}$, S. germaini $\mathrm{E}$, Conomyrma pogonius $^{\mathrm{E}}$, C. antarcticus $^{\mathrm{P}}$

Linepithema humile*

Camponotus morosus $\mathrm{E}$
Se compara el nivel de reconocimiento entre obreras pertenecientes a colonias artificiales (laboratorio) y naturales (terreno)

Se documenta el potencial efecto de esta especie como vector intrahospitalario de bacterias mesófilas C. morosus $\mathrm{E}^{\mathrm{E}}$, Brachymyrmex giardil $\mathrm{E}$,

En un gradiente altitudinal del sector precordillerano de Chile central, se estudia la abundancia y distribución espacial de especies y nidos de hormigas

Se documenta el potencial efecto de esta especie como vector de microorganismos enteropatógenos en industrias de alimentos

En un sector precordillerano de Chile central, se estudia la distribución espacial de nidos de esta especie

En una localidad precordillerana de Chile central, se estudia la actividad diaria de obreras exploradoras, durante las estaciones de primavera y verano

En un área precordillerana de Chile central, se analiza la distribución espacial, asociación interespecífica y asociación con arbustos, de los nidos de estas especies

Se estudia el reconocimiento entre individuos conespecíficos, en nidos artificiales 
TABLA 1 (continuación)

\begin{tabular}{|c|c|c|c|}
\hline Referencia & Temática estudiada & Especie analizada & Observaciones \\
\hline Ipinza-Regla et al. (1992) & Nota técnica & Camponotus morosus $\mathrm{E}$ & $\begin{array}{l}\text { Se analiza una técnica de revelado fotográfico } \\
\text { para marcaje de individuos }\end{array}$ \\
\hline Ipinza-Regla et al. (1993a) & Trayectorias de movimiento & Camponotus morosus $\mathrm{E}$ & $\begin{array}{l}\text { En un área precordillerana de Chile central, se } \\
\text { determinan la distancia recorrida, forma de } \\
\text { movimiento y ámbito de hogar de esta especie }\end{array}$ \\
\hline Ipinza-Regla et al. (1993b) & Reconocimiento entre individuos & Camponotus morosus $\mathrm{E}$ & $\begin{array}{l}\text { Se estudia el efecto de la distancia geográfica } \\
\text { entre colonias sobre la conducta de } \\
\text { reconocimiento entre obreras de esta especie }\end{array}$ \\
\hline Ipinza-Regla et al. (1994) & Reconocimiento entre individuos & $\begin{array}{l}\text { Camponotus morosus }{ }^{\mathrm{E}}, \\
\text { Brachymyrmex giardii }\end{array}$ & $\begin{array}{l}\text { Se analiza el nivel de "hermetismo" presente en las } \\
\text { conductas de reconocimiento entre individuos } \\
\text { homo y hetero específicos }\end{array}$ \\
\hline Ipinza-Regla et al. (1996) & Reconocimiento entre individuos & $\begin{array}{l}\text { Camponotus morosus } \mathrm{E}^{\mathrm{E}} \text {, } \\
\text { C. } \text { chilensis }^{\mathrm{P}} \text {, Solenopsis gayi } \mathrm{E}\end{array}$ & $\begin{array}{l}\text { Se determina la influencia de la distancia } \\
\text { filogenética sobre las conductas de } \\
\text { reconocimiento entre los individuos de estas } \\
\text { especies }\end{array}$ \\
\hline Ipinza-Regla et al. (1998) & Reconocimiento entre indivduos & Camponotus morosus $E$ & $\begin{array}{l}\text { Se analiza en terreno la conducta "hermetica" de } \\
\text { nidos de esta especie, frente a individuos intrusos } \\
\text { homoespecíficos dispuestos a diferentes } \\
\text { distancias de la entrada del nido }\end{array}$ \\
\hline Larraín et al. (1995) & Daño agrícola & Solenopsis gayi $E$ & $\begin{array}{l}\text { Se documenta el daño producido por esta especie, } \\
\text { a árboles nuevos de mandarinos y plantas de } \\
\text { pepino dulce }\end{array}$ \\
\hline Lewis \& Peredo (1993) & Uso del hábitat & Camponotus morosus $\mathrm{E}$ & $\begin{array}{l}\text { Se documenta la presencia de esta especie en } \\
\text { galerías abandonadas del "taladrador de la } \\
\text { madera" (Lepidoptera) en bosques de raulí }\end{array}$ \\
\hline
\end{tabular}

Medel \& Vásquez (1994)

Medel (1995)

Medel \& Fuentes (1995)

Moroni (1977)

Porter (1923b)

Ríos-Aramayo \& Jiménez (2000)

Ripa (1987)
Períodos de actividad, conductas Conomyrma goetschi ${ }^{\mathrm{E}}$, Brachymyrmex tróficas, patrones comunitarios giardil ${ }^{\mathrm{E}}$, Solenopsis gayl $\mathrm{E}^{\mathrm{E}}$

Conductas tróficas, patrones comunitarios

Períodos de actividad, conductas Pogonomyrmex vermiculatus ${ }^{\mathrm{P}}$, tróficas, patrones comunitarios, Solenopsis gayi $\mathrm{E}$ trayectorias de movimiento

Depredación/Perturbación Solenopsis gayi $\mathrm{E}$

Taxonomía

Períodos de actividad, conductas tróficas

Daño agrícola
Conomyrma goetschi $\mathrm{E}^{\mathrm{E}}$, Brachymyrmex giardil $\mathrm{E}$, Solenopsis gayi $\mathrm{E}$

Solenopsis gayl $\mathrm{E}^{\mathrm{E}}$, S. patagonica $\mathrm{P}^{\mathrm{P}}$, Tapinoma antarcticum $\mathrm{E}$

Camponotus hellmichi $\mathrm{E}^{\mathrm{E}}$

Estudio comparativo entre ensambles de hormigas granívoras presentes en zonas áridas de Chile y desierto del Monte en Argentina, donde se analizan: actividad de hormigas en el rango diario de temperaturas del suelo, distancia y tipo de forrajeo (individual o columnar)

Se examina el efecto de procesos históricos y de convergencia, sobre los patrones ecológicos de ensambles de hormigas granívoras presentes en Australia, Norte América y Sur América; se analizan factores como: abundancia de hormigas en cebos, distancia y tipo de forrajeo

En una localidad semiárida del norte de Chile, se documenta la actividad y forma de movimiento individual, dieta, y abundancia poblacional de estas especies

Se documenta la presencia de esta hormiga en estómagos de Pitius pitius (Ave)

Se documenta el diagnóstico de estas especies efectuado por parte de Santschi

Se estudia el número de visitas y la conducta de alimentación de estas hormigas, en cactus (Echinopsis chilensis) sin infestación e infestados por muérdagos (Tristerix aphyllus) y/0 conchuelas (Saissetia oleae)

Se discute el efecto protector de esta hormiga hacia insectos plagas (pulgones, conchuelas, chanchitos y mosquitas blancas) de árboles frutales 
TABLA 1 (continuación)

\begin{tabular}{|c|c|c|c|}
\hline Referencia & Temática estudiada & Especie analizada & Observaciones \\
\hline Ripa et al. (1993a) & Daño agrícola & Linepithema humile* & $\begin{array}{l}\text { Se evalua la acción de diferentes tratamientos } \\
\text { con insecticidas para controlar la "hormiga } \\
\text { argentina", lo cual permite el manejo del } \\
\text { "chanchito blanco" en cultivos de vid }\end{array}$ \\
\hline Ripa et al. (1993b) & Daño agrícola & Linepithema humile* & $\begin{array}{l}\text { Se analizan distintas técnicas de exclusión de } \\
\text { esta especie a cítricos, y de esta forma el manejo } \\
\text { de insectos plagas }\end{array}$ \\
\hline Ripa et al. (1998) & Daño agrícola & Linepithema humile*, Solenopsis gayi $\mathrm{E}$ & $\begin{array}{l}\text { Se revisa los daños directos e indirectos } \\
\text { producidos por estas especies a la agricultura, y } \\
\text { se discute la eficiencia de diferentes métodos de } \\
\text { control de estas hormigas }\end{array}$ \\
\hline Saiz \& Carvajal (1990) & Depredación/Perturbación & 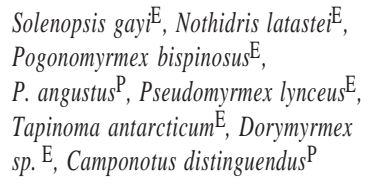 & $\begin{array}{l}\text { En el sector de Ocoa (V región), se evalúa el } \\
\text { efecto inmediato - y durante los dos años } \\
\text { siguientes - de una perturbación de fuego, sobre } \\
\text { la abundancia poblacional y capacidad de } \\
\text { recuperación de estos formícidos }\end{array}$ \\
\hline Snelling (1975) & Taxonomía & $\begin{array}{l}\text { Nothidris cekalovici } \mathrm{E}^{\mathrm{E}} \text {, Solenopsis } \\
\text { dysderces }^{\mathrm{E}} \text {, Dorymyrmex agallardo } \mathrm{E}, \\
\text { Conomyrma hunt } \mathrm{E}^{\mathrm{E}}, \text { C. hypocritus } \mathrm{E} \text {, } \\
\text { C. incomptus }{ }^{\mathrm{E}}, \text { C. pappodes }{ }^{\mathrm{E}}, \text { C. pogoniu }\end{array}$ & $\begin{array}{l}\text { Se documenta la descripción de estas nuevas } \\
\text { especies } \\
u s^{\mathrm{E}}\end{array}$ \\
\hline Solervicens et al. (1991) & Uso del hábitat & 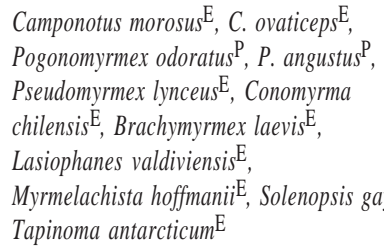 & $\begin{array}{l}\text { Durante la estación de primavera y en laderas de } \\
\text { exposición norte y sur, se estudia la entomofauna } \\
\text { de follaje y de superficie de suelo en la Reserva } \\
\text { Río Clarillo (región Metropolitana) } \\
u_{y i} \mathrm{E}^{\mathrm{E}} \text {, }\end{array}$ \\
\hline
\end{tabular}

En otro ámbito, sobresalen trabajos relacionados con ejes que definen el nicho ecológico en animales; esencialmente éstos corresponden a nueve artículos referidos al uso del hábitat (Ipinza 1971b, Ipinza-Regla \& Covarrubias 1982, IpinzaRegla et al. 1983, 1986, Covarrubias et al. 1987, Ipinza-Regla \& Covarrubias 1989, Ipinza-Regla et al. 1990, Solervicens et al. 1991, Lewis \& Peredo 1993); ocho estudios sobre períodos de actividad (Ipinza-Regla 1969, 1971 a, Hunt 1974, Ipinza-Regla et al. 1988, Medel \& Vásquez 1994, Medel \& Fuentes 1995, Fuentes et al. 1996, RíosAramayo \& Jiménez 2000); y siete publicaciones relacionadas a conductas tróficas de las hormigas (Grez et al. 1986, Medel \& Vásquez 1994, Medel 1995, Medel \& Fuentes 1995, Vásquez et al. 1995, Fuentes et al. 1996, Ríos-Aramayo \& Jiménez 2000). Muy relacionado al último tópico, figuran cinco estudios comunitarios sobre patrones ecológicos de ensambles de hormigas granívoras (Medel \& Vásquez 1994, Medel 1995, Medel \& Fuentes 1995, Vásquez et al. 1995, Fuentes et al. 1996), y cuatro trabajos que anali- zan la trayectoria de los movimientos de forrajeo exhibidos por obreras entre el nido de la colonia y sus fuentes de alimento (Goetsch 1930a, 1930b, Ipinza-Regla et al. 1993a, Medel \& Fuentes 1995).

Por otra parte, también se publicaron seis artículos sobre conductas de reconocimiento entre individuos (Ipinza-Regla et al. 1991, 1993b, 1994, 1996, 1998, Ipinza-Regla \& Morales 1998), cinco trabajos sobre daños producidos a árboles frutales (Ripa 1987, Ripa et al. 1993a, 1993b, Larraín et al. 1995, Ripa et al. 1998), y cuatro artículos sobre el efecto de la depredación y/o perturbación sobre distintas especies de formícidos (Moroni 1977, Fuentes \& Ipinza 1979, Ipinza-Regla 1985, Sáiz \& Carvajal 1990). Finalmente, cuatro trabajos analizaron el papel de las hormigas como vectores de enfermedades y/o productores de cuadros anafilácticos (Ipinza \& Capurro 1972, Ipinza \& Schenone 1972, Ipinza-Regla et al. 1981, 1984), y tres artículos fueron clasificados en la categoría de varios (Ipinza-Regla et al. 1992, Eaton \& Medel 1994, Córdova-Yamauchi et al. 1998). 
Géneros de formícidos en los que se ha concentrado la investigación

Los principales géneros de hormigas chilenas en los cuáles se realizaron estudios durante el siglo pasado se muestran en la Fig. 3 y Tabla 1. En este catastro no se incluyeron los trabajos que constituyen claves y/o listas taxonómicas de especies de formícidos (i.e., Goetsch 1933, Kusnezov 1959, 1963, Kempf 1970, 1972, Snelling \& Hunt 1975). Los resultados muestran que en 22 artículos existe información referida al género cosmopolita Camponotus (Ipinza 1971b, Fuentes \& Ipinza 1979, Ipinza-Regla et al. 1983, Ipinza-Regla 1985, Grez et al. 1986, Ipinza-Regla et al. 1986, Covarrubias et al. 1987, Ipinza-Regla et al. 1988, 1990, Sáiz \& Carvajal 1990, Ipinza-Regla et al. 1991, 1992, 1993a, 1993b, Solervicens et al. 1991, Lewis \& Peredo 1993, Eaton \& Medel 1994, Ipinza-Regla et al. 1994, 1996, 1998, IpinzaRegla \& Morales 1998, Ríos-Aramayo \& Jiménez 2000), y que en 20 trabajos se abordan aspectos biológicos del género Solenopsis (Porter 1923, Goetsch 1930a, 1930b, Ipinza-Regla 1969, 1971b, Snelling 1975, Moroni 1977, Ipinza-Regla \& Covarrubias 1982, Ipinza-Regla et al. 1983, Covarrubias et al. 1987, Ipinza-Regla et al. 1990,

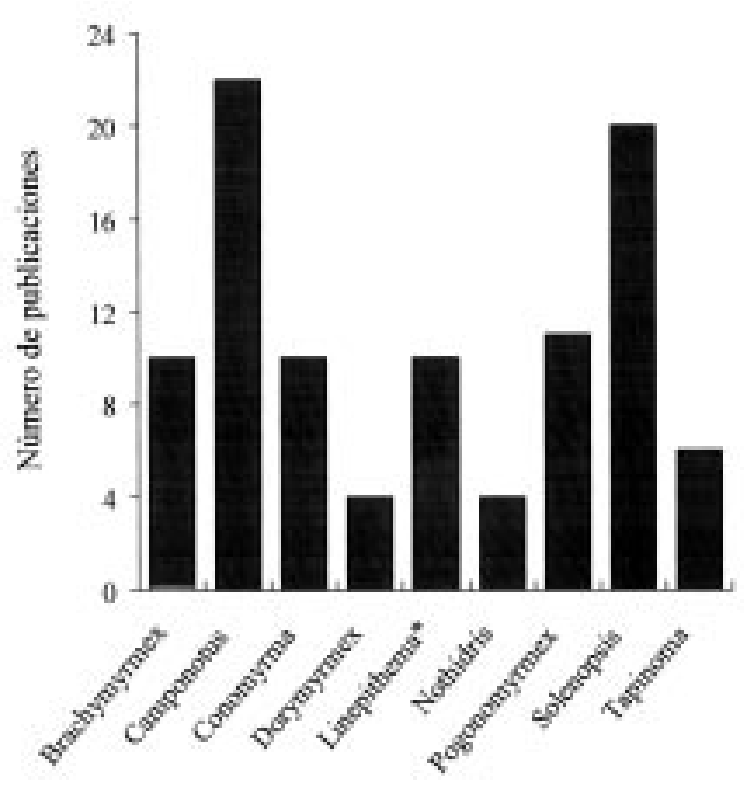

Género

Fig. 3: Géneros de hormigas chilenas en los cuáles se ha concentrado la investigación durante los últimos cien años. El símbolo (*) identifica géneros cuyas especies son introducidas.

Chilean ant genera on which investigation has been concentrated during the last hundred years. The (*) symbol indicates genera with introduced species.
Sáiz \& Carvajal 1990, Solervicens et al. 1991, Medel \& Vásquez 1994, Medel 1995, Medel \& Fuentes 1995, Larraín et al. 1995, Fuentes et al. 1996, Ipinza-Regla et al. 1996, Ripa et al. 1998); constituyendo éstos los dos principales géneros de hormigas en los cuales se ha concentrado la investigación.

En una siguiente categoría de importancia, aparecen el género Pogonomyrmex con 11 publicaciones (Goetsch 1930a, 1930b, Cekalovic 1964, Ipinza 1971b, Ipinza-Regla \& Covarrubias 1982, Ipinza-Regla et al. 1983, Ipinza-Regla 1985, Covarrubias et al. 1987, Sáiz \& Carvajal 1990, Solervicens et al. 1991, Medel \& Fuentes 1995), el género Brachymyrmex con 10 artículos (Ipinza 1971b, Ipinza-Regla et al. 1983, Ipinza-Regla 1985, Covarrubias et al. 1987, Ipinza-Regla et al. 1990, Solervicens et al. 1991, Ipinza-Regla et al. 1994, Medel \& Vásquez 1994, Medel 1995, Fuentes et al. 1996), y el género Conomyrma también con 10 trabajos (Hunt 1974, Snelling 1975, IpinzaRegla et al. 1983, Covarrubias et al. 1987, IpinzaRegla \& Covarrubias 1989, Ipinza-Regla et al. 1990, Solervicens et al. 1991, Medel \& Vásquez 1994, Medel 1995, Fuentes et al. 1996). En otro orden, existe información del género Linepithema* en 10 artículos (Ipinza 1971b, Ipinza-Regla et al. 1981, 1984, Ipinza-Regla 1985, Ripa 1987, Ipinza-Regla \& Covarrubias 1989, Ripa et al. 1993a, 1993b, 1998, CórdovaYamauchi et al. 1998), antecedentes del género Tapinoma en seis publicaciones (Porter 1923, Ipinza 1971b, Hunt 1974, Ipinza-Regla \& Covarrubias 1989, Sáiz \& Carvajal 1990, Solervicens et al. 1991), datos sobre el género Nothidris en cuatro trabajos (Ettershank 1965, Snelling 1975, Ipinza-Regla \& Covarrubias 1982, Sáiz \& Carvajal 1990), e información sobre el género Dorymyrmex también en cuatro artículos (Ipinza 1971b, Snelling 1975, Ipinza-Regla \& Covarrubias 1989, Sáiz \& Carvajal 1990).

Finalmente, existen antecedentes biológicos en tres publicaciones para el género Myrmelachista (Ipinza 1971b, Ipinza-Regla 1985, Solervicens et al. 1991), datos en un par de trabajos para los géneros Antichthonidris (Snelling 1975, IpinzaRegla \& Covarrubias 1982), Heteroponera (Ipinza \& Capurro 1972, Ipinza \& Schenone 1972), Pseudomyrmex (Sáiz \& Carvajal 1990, Solervicens et al. 1991), y Tetramorium* (Ipinza-Regla \& Covarrubias 1982, Ipinza-Regla 1985); y alguna información traza presente en un artículo para los géneros Lasiophanes (Solervicens et al. 1991), Monomorium* (Ipinza-Regla \& Covarrubias 1982), y Pheidole (Ipinza-Regla \& Covarrubias 1982). 


\section{DISCUSIÓN}

Durante los primeros tres períodos del siglo XX (1900-1929) la información existente respecto a hormigas estuvo basada principalmente en el reporte de pequeñas notas sobre la vida de hormigas foráneas. La Revista Chilena de Historia Natural fue el principal órgano de difusión de este tipo de información, lo cual fue promovido esencialmente a través de su editor C. Porter, el cual tendría además una fuerte injerencia en el impulso y desarrollo de la ciencia y entomología en Chile (González 1983, Etcheverry 1992). A pesar de los avances conseguidos durante estos años en el cultivo de las ciencias naturales (González 1983), la nula productividad nacional respecto a la temática aquí tratada puede haber estado ligada a una visión de época en que "la ciencia sólo se adquiere a través del discurso o los libros" y/o la existencia de "una reverenciosa sumisión ante la abrumadora presencia de la capacidad creadora de la ciencia europea, unida a una pesimista apreciación del vuelo intelectual o inventivo de los chilenos" (Croxatto 1982). Otra razón que ha sido sugerida como causa de esta baja producción en las ciencias naturales, son las restricciones presupuestarias y una reducción de la planta de investigadores que afectó al Museo Nacional de Historia Natural durante esta época (Veloso 1982).

En la década del 30' se produce un importante auge en la generación de información sobre hormigas chilenas, lo cual esta ligado esencialmente al nombre de W. Goetsch, el cual elaboró en solitario y en coautoría con C. Menozzi catálogos y listas de especies de los formícidos presentes en Chile. Además, este investigador desarrolló una serie de experimentos destacados e ingeniosos para su época, en los cuáles analizaba el rol de la comunicación química sobre las conductas de movimientos exhibidas por obreras entre el nido y una fuente de alimento, aspectos que están plenamente vigentes en líneas de investigación desarrolladas en la actualidad (Holldobler \& Wilson 1990). Posteriormente, las décadas del 40' y 50' son percibidas como un período de nula productividad en la temática tratada, lo cual puede haber estado determinado por el cese de C. Porter en sus funciones de editor de la Revista Chilena de Historia Natural en 1940 y su posterior fallecimiento en 1942 (González 1983), lo cual habría tenido un profundo impacto en el auge que venía experimentando la entomología en el país. A esto hay que agregar, que hasta fines de los años 40' el cultivo de las ciencias naturales en Chile seguía siendo de orden clásico, es decir, una disciplina descriptiva y comparativa, mientras que a nivel mundial lo que imperaba era la formación de científicos especializados en investigar las distintas disciplinas en que se subdividían las ciencias naturales; todo lo cual generaba el siguiente escenario "existía en Chile un período de suspenso en cuanto al rumbo a seguir y los museos se preocupaban más de conservar que de investigar" (González 1983).

A partir de la década de los 60' se produce una revitalización de la producción científica en el tema tratado, lo cual parece estar ligado a una canalización de la enseñanza e investigación de las ciencias naturales en el ambiente de la universidad. Específicamente las actividades desarrolladas por J. Herrera en el Centro de Estudios Entomológicos, G. Mann en el Centro de Investigaciones Zoológicas, y la creación por parte de J. Gómez Millas de la Facultad de Ciencias de la Universidad de Chile, dieron un fuerte impulso al cultivo de disciplinas como la zoología y ecología (Croxatto 1982, Hajek 1982, Veloso 1982, González 1983). Durante la década del 70' la elaboración de la tesis doctoral de J.H. Hunt (1973) - inmersa en un estudio de convergencia ecológica entre ecosistemas mediterráneos de Chile y California, dependiente del "International Biological Program" (IBP) - constituye un hito destacado en el que se analiza y compara la ecología de comunidades de hormigas en estas regiones, recopilándose información básica sobre la biología de varias especies de formícidos. La importancia de este tipo de información alcanza su máxima expresión con la publicación del valioso catálogo acerca de las hormigas de Chile por parte de Snelling \& Hunt (1975), el cual es utilizado en la actualidad para la identificación y determinación de organismos obreras a nivel de subfamilias, géneros y especies. Paralelamente, durante estos años aparecen textos clásicos (Wilson 1971, Oster \& Wilson 1978) que incentivarán y fomentarán a nivel nacional el estudio biológico de estos insectos sociales.

En la década de los 80', sobresalen los trabajos de Ipinza-Regla y colaboradores, los cuáles se concentran en estudiar especies del género Camponotus abordando una serie de temáticas ecológicas. La aparición continuada de la Revista Chilena de Entomología y la edición de una nueva revista - Acta Entomológica Chilena - durante estos años, juegan un papel primordial en la divulgación de esta información. A pesar que el año 1983 se comienza a reeditar la Revista Chilena de Historia Natural, la aparición de información sobre hormigas chilenas ha sido prácticamente nula en esta publicación. En el libro "Introducción a los insectos de Chile" de Peña (1986) se hace una breve mención de dos páginas a las hormigas chilenas que presentan un amplio rango de distri- 
bución y resultan ser más comunes y/o abundantes; por otra parte, en la segunda edición en lengua castellana de la obra "La vida social de las hormigas" de Goetsch (1987) se documentan varios aspectos acerca de la biología de hormigas chilenas; adicionalmente, en otro texto (González 1989) se menciona la importancia económica de la "hormiga argentina" (Linepithema humile*) como plaga primaria. Finalmente, durante la década de los 90' se continúan y mejoran los aspectos desarrollados durante los años 80'; es así como a la producción continuada de Ipinza-Regla, se suman y destacan los trabajos de Medel y colaboradores sobre aspectos comunitarios de ensambles de hormigas granívoras. Además, durante este período en una serie de libros se entregan antecedentes acerca de las especies de hormigas que constituyen plagas secundarias (Prado 1991) y/o presentan algún interés económico (Artigas 1994), el estado del conocimiento de los formícidos chilenos (Rojas \& Elgueta 1995), y el daño producido por ciertas especies en cítricos (Ripa \& Rodríguez 1999). Por último, es importante mencionar que a comienzos de esta década se publicó por parte de Holldobler \& Wilson (1990) la monumental obra "The ants" - texto de consulta obligada para cualquier interesado en el tema - en la cual existe alguna información traza sobre Chile y sus hormigas.

En relación a las principales temáticas estudiadas, se puede afirmar que a partir de la década del 20' los trabajos sobre taxonomía fueron preponderantes y tuvieron una aparición periódica (salvo durante la década del 40'), culminando con la publicación del trabajo de Snelling \& Hunt (1975). Este fenómeno puede deberse a que cuando se comienza el estudio de un taxón, un primer e importante paso es conocer cuántas y qué tipo de especies existen. Sin embargo, en los últimos 25 años no se han publicado trabajos taxonómicos en hormigas chilenas, lo cual esta asociado a un patrón general de disminución de especialistas interesados en trabajar actualmente en esta temática (Simonetti 1995). De hecho, en Chile existen sólo cinco entomólogos nacionales que trabajan en forma regular en aspectos taxonómicos de Hymenoptera (Solervicens 1995), de los cuáles sólo uno (J. Ipinza-Regla) se especializa en Formicidae (Solervicens, comunicación personal). Un segundo y necesario paso en el estudio de un taxón, consiste en analizar aspectos básicos de la biología de los organismos; lo cual se corresponde con lo sucedido durante la década del $80^{\prime}$ y 90', donde han tenido una fuerte preponderancia artículos sobre la ecología (e.g., uso del hábitat, períodos de actividad, conductas tróficas) de las hormigas chilenas (e.g., trabajos de IpinzaRegla, Medel y colaboradores).
Respecto a los géneros de formícidos que han concentrado el interés de las investigaciones, no constituye un hecho sorprendente que los estudios se hayan focalizado principalmente en Camponotus y Solenopsis, debido a que estos géneros corresponden a hormigas que poseen un amplio rango de distribución y se presentan en forma común y abundante en el país (Snelling \& Hunt 1975, Peña 1986, Artigas 1994). Por otra parte, destacan los trabajos que se han centrado en géneros de hormigas granívoras (Conomyrma, Brachymyrmex, Pogonomyrmex), así como información reciente referida a los efectos y consecuencias como plaga que tendría la especie Linepithema humile* (González 1989, Prado 1991, Artigas 1994, Ripa \& Rodríguez 1999). Algunas características conspicuas de estas especies como su tamaño corporal, coloración, tipo de nido, nivel de actividad, forma de movimiento, y conducta de alimentación, sumado al hecho de su presencia en Chile central (donde se ha concentrado la investigación), las convierten en objetos preferidos de estudio.

Es posible que existan datos marginales sobre biología de hormigas chilenas en otros artículos; sin embargo, los antecedentes aquí revisados corresponden a los trabajos más representativos y que de alguna forma dan cuenta del estado del conocimiento de este grupo de organismos. Se espera que esta información pueda contribuir a biólogos interesados en estudiar a las hormigas y su vida social, así como ayude a fomentar la publicación de datos y tesis de investigadores que han trabajado en el tema. Algunas carencias generales que han sido detectadas en el conocimiento de los insectos de Chile, se proyectan en el estudio de estos organismos. Es así como se hace necesario fomentar la formación de taxónomos y sistemáticos especializados en este grupo, complementar y enriquecer las colecciones taxonómicas, incentivar su estudio no sólo en localidades de Chile central sino también en regiones del norte y sur del país (i.e., análisis biogeográficos), y acceder a información básica referente a los ciclos de vida y ecología de estos organismos (Solervicens 1995). El énfasis de estas tareas debiera ser puesto en las hormigas endémicas, debido a su importancia e implicancia en problemas de biodiversidad y conservación de especies. Las hormigas chilenas constituyen un excelente modelo de estudio para desarrollar varias líneas de investigación. En el ámbito de la ecología conductual de individuos, sería posible la complementación de cuerpos de conocimiento tan diversos como: forrajeo social o en grupo, navegación espacial, uso de información, y comunicación química entre obreras. Los análisis 
de proporción de sexos y castas, sobrevivencia y reproducción de nidos, estructura y variabilidad genética de las colonias, permitirían abordar problemáticas de la biología de poblaciones. El estudio de las interacciones entre especies de hormigas y con otros insectos y plantas, así como la partición del hábitat y de la actividad temporal por parte de formícidos, incrementaría la comprensión de la estructura comunitaria de los ensambles de hormigas. Finalmente, el análisis de la remoción y mezcla de sustrato por parte de la fauna mirmecológica, permitiría determinar su rol e impacto en funciones y procesos ecosistémicos. La recopilación de toda esta información posibilitaría abordar preguntas como ¿existen diferentes y complejas estrategias adaptativas cuando obreras y/o colonias se ven confrontadas a ambientes espacial y temporalmente variables?, y evaluar sus implicancias en el ámbito de la ecología y biología evolutiva de insectos sociales.

\section{AGRADECIMIENTOS}

Considero importante destacar la enorme utilidad de los catálogos sobre revistas científicas nacionales elaborados y publicados por M. Etcheverry en la Revista Chilena de Historia Natural, los cuáles incentivaron y guiaron el comienzo de esta revisión. Por otra parte, agradezco a J. Solervicens del Instituto de Entomología de la Universidad Metropolitana de Ciencias de la Educación, a F. Rojas, A. Camousseight y M. Elgueta de la Sección Entomología del Museo Nacional de Historia Natural, y al bibliotecario jefe de este museo L. Hidalgo, quienes permitieron el acceso a la colección de revistas en las bibliotecas de ambas instituciones. Javier Simonetti facilitó literatura que enriqueció la discusión de este trabajo, y J. IpinzaRegla proveyó de artículos de difícil disponibilidad. Agradezco especialmente a R. Vásquez y R. Medel por la lectura crítica de una versión preliminar de este manuscrito. Finalmente, destacó los comentarios y la fluidez con que este artículo fue revisado por parte de dos revisores anónimos y por el editor asociado a este trabajo. Hugo TorresContreras es becario doctoral CONICYT. Este trabajo fue financiado parcialmente por Proyecto FONDECYT Doctorado 2010001.

\section{LITERATURA CITADA}

ARTIGAS JN (1994) Entomología Económica: insectos de interés agrícola, forestal, médico y veterinario. Volumen 1. Ediciones Universidad de Concepción, Concepción, Chile. 1126 pp.
BERG C (1890) Enumeración sistemática y synonimica de los Formicidos argentinos, chilenos y uruguayos. Anales de la Sociedad Científica Argentina (Buenos Aires) 29: 5-43.

BOURKE AFG (1997) Sociality and kin selection in insects. En: Krebs JR \& NB Davies (eds) Behavioural ecology: an evolutionary approach: 203-227. Blackwell Science Ltd., Oxford, United Kingdom.

BROCKMANN HJ (1984) The evolution of social behaviour in insects. En: Krebs JR \& NB Davies (eds) Behavioural ecology: an evolutionary approach: 340361. Blackwell Scientific Publications, Oxford, United Kingdom.

CAMPOS L \& L PEÑA (1973) Los insectos de Isla de Pascua. Revista Chilena de Entomología 7: 217-229.

CEKALOVIC T (1964) Las hormigas chilenas del género Pogonomyrmex Mayr (Hymenoptera - Formicidae) y su hallazgo en Magallanes (Chile). Noticiario Mensual del Museo Nacional de Historia Natural (Chile) 100 (noviembre): s/p.

CORDOVA-YAMAUCHI L, E GIANOLI, A QUIRÓZ \& H NIEMEYER (1998) The argentine ant, Linepithema humile (Hymenoptera: Formicidae: Dolichoderinae) is sensitive to semiochemicals involved in the spacing behaviour in the bird cherry-oat aphid Rhopalosiphum padi (Sternorrhyncha: Aphididae). European Journal of Entomology 95: 501-508.

COVARRUBIAS R, R FUEYO \& J IPINZA (1987) Algunos factores ecológicos que influencian la distribución espacial de nidos de hormigas. Acta Entomológica Chilena 14: 117-126.

CROXATTO HR (1982) Las ciencias biológicas en Chile. En: Academia de Ciencias, Instituto de Chile \& Corporación de Promoción Universitaria (eds) Una visión de la comunidad científica nacional; las actividades de investigación y desarrollo en Chile: 83-107. Santiago, Chile.

EATON LC \& RG MEDEL (1994) Allozyme variation and genetic relatedness in a population of Camponotus chilensis (Hymenoptera: Formicidae) in Chile. Revista Chilena de Historia Natural 67: 157-161.

EMERY C (1894) Notes sur les fourmis du Chili. Avec descriptions de duex espèces nouvelles. Actas de la Societé Scientifique du Chili 4: 213-216.

EMERY C (1895) Duexieme note sur les fourmis de Chili. Actas de la Societé Scientifique du Chili 5: 10-18.

ETTERSHANK G (1965) A new species of Megalomyrmex from the Chilean Andes (Formicidae, Hymenoptera). Psyche 72: 55-58.

FOWLER HG (1981) Behavior of two myrmecophiles of paraguayan leaf-cutting ants. Revista Chilena de Entomología 11: 69-72.

FOWLER HG (1988) A organizacao das comunidades de formigas no estado de mato grosso, Brasil. Anales del Museo de Historia Natural de Valparaíso (Chile) 19: 35-42.

FUENTES ER \& J IPINZA (1979) A note on the diet of Liolaemus monticola (Reptilia, Lacertilia, Iguanidae). Journal of Herpetology 13: 121-123.

FUENTES JE, S HERRERA \& RG MEDEL (1996) Observaciones preliminares sobre uso de recursos y temperatura de actividad en ensambles de hormigas granívoras del norte de Chile. Acta Entomológica Chilena 20: 13-17. 
GALLARDO A (1908) De como se fundan los nuevos hormigueros de hormiga negra. Revista Chilena de Historia Natural 12: 27-31.

GOETSCH W (1930a) Como se entienden entre si las hormigas. Revista del Instituto Bacteriológico de Chile 1: $11-19$.

GOETSCH W (1930b) Observaciones y experimentos con animales chilenos: medios de comunicación entre los socios y división del trabajo en los nidos de las hormigas. Anales de la Universidad de Chile 8: 979-1018.

GOETSCH W (1933) Formicidae chilensis (Hormigas chilenas). Boletín de la Sociedad de Biología de Concepción (Chile) 7: 11-28.

GOETSCH W (1987) La vida social de las hormigas. Segunda edición. Editorial Labor/Punto Omega, Barcelona, España. 218 pp.

GOETSCH W \& C MENOZZI (1935) Die ameisen Chiles. Konowia 14: 95-102.

GONZÁLEZ RH (1983) Desarrollo de las Ciencias Naturales en Chile. En: Cuadernos de la Universidad de Chile No. 2. Conferencias del ciclo: el desarrollo de la ciencia en Chile y sus perspectivas futuras: 87-110. Editorial Universitaria, Santiago, Chile.

GONZÁLEZ RH (1989) Insectos y ácaros de importancia agrícola y cuarentenaria en Chile. Editorial Ograma, Santiago, Chile. 310 pp.

GORDON DM (1996) The organization of work in social insects colonies. Nature 380: 121-124.

GREZ AA, JA SIMONETTI \& JH IPINZA-REGLA (1986) Hábitos alimenticios de Camponotus morosus (Smith, 1858) (Hymenoptera: Formicidae) en Chile central. Revista Chilena de Entomología 13: 51-54.

HAJEK ER (1982) La ecología en Chile. En: Academia de Ciencias, Instituto de Chile \& Corporación de Promoción Universitaria (eds) Una visión de la comunidad científica nacional; las actividades de investigación y desarrollo en Chile: 138-150. Santiago, Chile.

HAMILTON WD (1964) The genetical evolution of social behaviour. Journal of Theoretical Biology 7: 1-52.

HEINRICH B (1978) The economics of insect sociality. En: Krebs JR \& NB Davies (eds) Behavioural ecology: an evolutionary approach: 97-128. Blackwell Scientific Publications, Oxford, United Kingdom.

HOLLDOBLER B \& EO WILSON (1990) The ants. The Belknap Press of Harvard University Press, Cambridge, Massachusetts. 732 pp.

HUNT JH (1973) Comparative ecology of ant communities in mediterranean regions of California and Chile. Ph.D. Dissertation, University of California, Berkeley. $131 \mathrm{pp}$.

HUNT JH (1974) Temporal activity patterns in two competing ant species (Hymenoptera: Formicidae). Psyche 81: 237-242.

IPINZA-REGLA J (1969) Las hormigas como organismos recuperadores en la sabana espinosa chilena. Noticiario Mensual del Museo Nacional de Historia Natural (Chile) 153: 13-14.

IPINZA-REGLA J (1971a) Presencia de Formicoidea en la sabana climax espinosa de Chile (estrato arbóreo-arbustivo). Revista Peruana de Entomología 14: 346351.

IPINZA-REGLA J (1971b) Importancia de formícidos en sabana espinosa central de Chile (estrato arbóreoarbustivo. Curacaví). Boletín de la Universidad de Chile 113: 25-31
IPINZA-REGLA JH (1985) Formícidos en el contenido gástrico de Liolaemus monticola (Reptilia). Revista Chilena de Entomología 12: 165-168.

IPINZA J \& L CAPURRO (1972) Heteroponera carinifrons en Antofagasta. Noticiario Mensual del Museo Nacional de Historia Natural (Chile) 192: 7.

IPINZA J \& H SCHENONE (1972) Heteroponera carinifrons, hormiga causante de cuadros anafilácticos en Chile. Boletín Chileno de Parasitología 27: 57-58.

IPINZA-REGLA JH \& R COVARRUBIAS (1982) Distribución de especies de la subfamilia Myrmicinae Formicidae en Chile. Revista Española de Entomología 58: 135-141.

IPINZA-REGLA J \& R COVARRUBIAS (1989) Répartition des espèces de Dolichoderinae (Hymenoptera: Formicidae) au Chili. Annales de la Societé Entomologique de France 25: 377-379.

IPINZA-REGLA J \& MA MORALES (1998) Hermetismo en laboratorio y condiciones naturales para Camponotus morosus Smith, 1858 (Hymenoptera, Formicidae). Gayana Zoología (Chile) 62: 177-181.

IPINZA-REGLA J, G FIGUEROA \& J OSORIO (1981) Iridomyrmex humilis "hormiga argentina" como vector de infecciones intrahospitalarias. I.- Estudio Bacteriológico. Folia Entomológica Mexicana 50: 81-96.

IPINZA-REGLA JH, R COVARRUBIAS \& R FUEYO (1983) Distribución altitudinal de Formicidae en los andes de Chile central. Folia Entomológica Mexicana 55: 103-128

IPINZA-REGLA J, G FIGUEROA \& I MORENO (1984) Iridomyrmex humilis (Formicidae) y su papel como posible vector de contaminación microbiana en industrias de alimentos. Folia Entomológica Mexicana 62: 111-124

IPINZA-REGLA JH, MA MORALES, A LUCERO, P PLÁ, L VALENCIA \& J MARTÍNEZ (1986) Distribución espacial de nidos de Camponotus morosus (Smith, 1858) Formicidae, en un ambiente precordillerano de Chile central. Boletín de la Sociedad de Biología de Concepción (Chile) 57: 75-79.

IPINZA-REGLA JH, R COVARRUBIAS, MA MORALES, A MANN, J MARTÍNEZ, P PLÁ \& A RIVERA (1988) Períodos de actividad de Camponotus morosus (Smith, 1858), (Formicidae). Medio Ambiente (Chi1e) 9: 57-64

IPINZA-REGLA JH, R COVARRUBIAS, MA MORALES (1990) Distribución espacial de nidos de hormigas en un área precordillerana de Chile central. Folia Entomológica Mexicana 79: 163-174.

IPINZA-REGLA JH, A LUCERO \& MA MORALES (1991) Hermetismo en sociedades de Camponotus morosus Smith, 1858 (Hymenoptera, Formicidae) en nidos artificiales. Revista Chilena de Entomología 19: 29-38.

IPINZA-REGLA J, J MARTÍNEZ \& J SOLORZA (1992) Marcaje de Camponotus morosus Smith, 1858 (Hymenoptera: Formicidae) según la técnica de Fresneau y Charpin. Acta Entomológica Chilena 17: 237-239.

IPINZA-REGLA J, A GALANO \& MA MORALES (1993a) Ambito de hogar de Camponotus morosus Smith, 1858 (Hymenoptera, Formicidae). Revista Chilena de Entomología 20: 23-30. 
IPINZA-REGLA JH, MA MORALES \& S SEPÚLVEDA (1993b) Hermetismo y distancia geográfica en sociedades de Camponotus morosus Smith, 1858 (Hymenoptera: Formicidae). Acta Entomológica Chilena 18: 127-132.

IPINZA-REGLA JH, C CARBONELL \& MA MORALES (1994) Hermetismo en sociedades mixtas de hormigas (Hymenoptera: Formicidae) en nidos artificiales. Revista Chilena de Entomología 21: 41-45.

IPINZA-REGLA JH, MA MORALES \& V AROS (1996) Hermetismo entre tres especies de hormigas. Boletín de la Sociedad de Biología de Concepción (Chile) 67: 33-36.

IPINZA-REGLA J, C NÚÑEZ \& MA MORALES (1998) Hermetismo de Camponotus morosus Smith, 1858 (Hymenoptera: Formicidae) en terreno. Folia Entomológica Mexicana 103: 55-61.

KEMPF WW (1970) Catálogo das formigas do Chile. Papéis Avulsos de Zoologia 23: 17-43.

KEMPF WW (1972) Correcao par o "Catalogo das formigas do Chile”. Studia Entomológica 15: 448.

KREBS JR \& NB DAVIES (1993) Behavioral ecology: an introduction to behavioural ecology. Blackwell Scientific Publications, Oxford, United Kingdom. 420 pp.

KUSCHEL G (1952) Introducción a "Los insectos de las islas Juan Fernández". Revista Chilena de Entomología 2: 3-6.

KUSNEZOV N (1959) La fauna de hormigas en el oeste de la Patagonia y Tierra del Fuego. Acta Zoológica Lilloana (Argentina) 17: 321-401.

KUSNEZOV N (1963) Zoogeografía de las hormigas en Sudamerica. Acta Zoologica Lilloana (Argentina) 19: 25-186.

LA REDACCIÓN (1914) Contra las hormigas. Anales de Zoología Aplicada 1: 87-88.

LARRAÍN P, J IPINZA-REGLA \& L ÁLVAREZ (1995) Daño de la hormiga Solenopsis gayi (Spinola) (Hymenoptera: Formicidae) a mandarinos (Citrus reticulata Blanco) y pepino dulce (Solanim muricatum Ait.) en la IV región. Agricultura Técnica (Chile) 55: 164-166.

LEWIS PD \& AA PEREDO (1993) Camponotus morosus (Smith) (Hymenoptera, Formicidae) en galerías abandonadas de Chilecomadia valdiviana (Philippi) (Lepidoptera, Cossidae) en Nothofagus alpina (Poepp. et Endl.) Oerst. Boletín de la Sociedad de Biología de Concepción (Chile) 64: 97-98.

MEDEL RG (1995) Convergence and historical effects in harvester ant assemblages of Australia, North America, and South America. Biological Journal of the Linnean Society 55: 29-44.

MEDEL RG \& RA VÁSQUEZ (1994) Comparative analysis of harvester ant assemblages of Argentinian and Chilean arid zones. Journal of Arid Environments 26: 363-371.

MEDEL RG \& JE FUENTES (1995) Notes on the individual activity, diet, and abundance of the ants Pogonomyrmex vermiculatus and Solenopsis gayi (Hymenoptera: Formicidae) in a semiarid ecosystem of northern Chile. Revista Chilena de Entomología 22: $81-84$

MENOZZI C (1935) La formiche del Chile. Zoologische Jahrbucher 67: 319-336.
MORONI J (1977) Solenopsis gayi (Hymenoptera, Formicidae) en la dieta de Pitius pitius (Aves, Picidae). Boletin del Museo Nacional de Historia Natural (Chile) $35: 83-85$

OSTER G \& EO WILSON (1978) Caste and ecology in the social insects. Princeton University Press, Princeton, New Jersey. 352 pp.

PEÑA LE (1986) Introducción a los insectos de Chile. Editorial Universitaria, Santiago, Chile. 256 pp.

PEÑA LE (1987) Consideraciones sobre la fauna de artrópodos terrestres de las Islas Oceánicas Chilenas. En: Castilla JC (ed) Islas oceánicas chilenas; conocimiento científico y necesidades de investigación: 217223. Ediciones Universidad Católica de Chile, Santiago, Chile.

PÉREZ-CANTO J (1937) La destrucción de las hormigas. Anales de la Academia Chilena de Ciencias Naturales 22: $125-126$.

PORTER CE (1910) Hongos cultivados por hormigas. Revista Chilena de Historia Natural 14: 111.

PORTER CE (1915) Larva que vive en simbiosis con hormigas. Revista Chilena de Historia Natural 19: 50.

PORTER CE (1923a) Bracónidos de los nidos de hormigas. Revista Chilena de Historia Natural 27: 194.

PORTER CE (1923b) Hormigas nuevas chilenas. Revista Chilena de Historia Natural 27: 196-197.

PORTER CE (1926) Hormigas contra piojos. Revista Chilena de Historia Natural 30: 401-402.

PRADO E (1991) Artrópodos y sus enemigos naturales asociados a plantas cultivadas en Chile. Instituto de Investigaciones Agropecuarias, Serie Boletín Técnico $\mathrm{N}^{\circ} 169$, Santiago, Chile. 207 pp.

RÍOS-ARAMAYO R \& M JIMÉNEZ (2000) Do armored scale insects influence visit rate and feeding activity of ants on Echinopsis chilensis. Revista Chilena de Entomología 26: 65-68.

RIPA R (1987) Las hormigas y el hombre. Investigación y Progreso Agropecuario, La Platina (Chile) 44: 52-54.

RIPA R \& F RODRÍGUEZ (1999) Plagas de cítricos, sus enemigos naturales y manejo. Colección Libros Instituto Nacional de Investigaciones Agropecuarias $\mathrm{N}^{\circ}$ 3. $151 \mathrm{pp}$.

RIPA R, F RODRÍGUEZ \& F ROJAS (1993a) Nuevos avances en el manejo del chanchito blanco de la vid: control de la hormiga y manejo de la canopia. Investigación y Progreso Agropecuario, La Platina (Chile) 76: 28-30.

RIPA R, S ROJAS \& F RODRÍGUEZ (1993b) Plagas más importantes de los cítricos y su manejo. II.- Acaro de la yema, escama roja, conchuela acanalada, pulgones, caracol de las viñas, control de hormigas, manejo de la poda. Investigación y Progreso Agropecuario, La Platina (Chile) 79: 5-11.

RIPA R, F RODRÍGUEZ \& M RUST (1998) Las hormigas y su relación con la agricultura. Revista Frutícola 19: 85-92.

ROJAS F \& M ELGUETA (1995) Hymenoptera. En: Simonetti JA, MTK Arroyo, AE Spotorno \& E Lozada (eds) Diversidad biológica de Chile: 280-298. Comisión Nacional de Ciencia y Tecnología, Santiago, Chile.

SAIZ F \& C CARVAJAL (1990) Incendios forestales en el parque nacional La Campana, sector Ocoa, V región, Chile. V.- Blattodea, Formicidae y Mutillidae. Impacto y recuperación. Anales del Museo de Historia Natural de Valparaíso (Chile) 21: 51-61. 
SANTSCHI F (1922) Descriptions de nouvelles fourmis de 1' Argentine et pays limitrophes. Anales de la Sociedad Científica Argentina (Buenos Aires) 94: 241-262.

SEGER J (1991) Cooperation and conflict in social insects. En: Krebs JR \& NB Davies (eds) Behavioural ecology: an evolutionary approach: 338-373. Blackwell Scientific Publications, Oxford, United Kingdom.

SHATTUCK SO (1992) Review of the Dolichoderine ant genus Iridomyrmex Mayr with descriptions of three new genera (Hymenoptera: Formicidae). Journal of the Australian Entomological Society 31: 13-18.

SIMONETTI JA (1995) Diversidad biológica: algo más que nombres, algo más que números. En: Simonetti JA, MTK Arroyo, AE Spotorno \& E Lozada (eds) Diversidad biológica de Chile: 1-4. Comisión Nacional de Ciencia y Tecnología, Santiago, Chile.

SNELLING RR (1975) Descriptions of new Chilean ant taxa (Hymenoptera: Formicidae). Los Angeles County, California Museum of Natural History, Contributions in Science 294: 1-19.

SNELLING RR (1981) Systematics of social Hymenoptera. En: Hermann HR (ed) Social insects: 369-474. Academic Press, New York, New York.

SNELLING RR \& JH HUNT (1975) The ants of Chile (Hymenoptera: Formicidae). Revista Chilena de Entomología 9: 63-129.

SOLERVICENS J (1995) Consideraciones generales sobre los insectos, el estado de su conocimiento y las colecciones. En: Simonetti JA, MTK Arroyo, AE Spotorno \& E Lozada (eds) Diversidad biológica de Chile: 198-210. Comisión Nacional de Ciencia y Tecnología, Santiago, Chile.
SOLERVICENS J, P ESTRADA \& M MÁRQUEZ (1991) Observaciones sobre entomofauna de suelo y follaje en la Reserva Nacional Río Clarillo, región metropolitana, Chile. Acta Entomológica Chilena 16: 161-182.

SPINOLA M (1851) Formicidae. En: Gay C (ed) Historia física y política de Chile, Zoología 6: 232-246. Imprenta de Maulde et Renou, Paris, Francia.

TRIVERS RL \& H HARE (1976) Haplodiploidy and the evolution of the social insects. Science 191:249-263.

VÁSQUEZ RA, RO BUSTAMANTE \& JA SIMONETTI (1995) Granivory in the Chilean matorral: extending the information on arid zones of South America. Ecography 18: 403-409.

VELOSO A (1982) La zoología terrestre. En: Academia de Ciencias, Instituto de Chile y Corporación de Promoción Universitaria (eds) Una visión de la comunidad científica nacional; las actividades de investigación y desarrollo en Chile: 190-204. Santiago, Chile.

WHEELER WM (1923) Formicidae from Easter Island and Juan Fernandez. En: Skosttberg C (ed) The Natural History of the Juan Fernández and Easter Island, Zoology 3: 317-319. Almqvist \& Wiksells Boktryckeri AB, Upsala, Sweden.

WILSON EO (1971) The insect societies. The Belknap Press of Harvard University Press, Cambridge, Massachusetts. 548 pp.

WILSON EO (1985) The sociogenesis of insect colonies. Science 228: 1489-1495.

WOLFFSOHN JA (1920) Las hormigas como huéspedes en las colmenas. Anales de Zoología Aplicada 7: 43. 